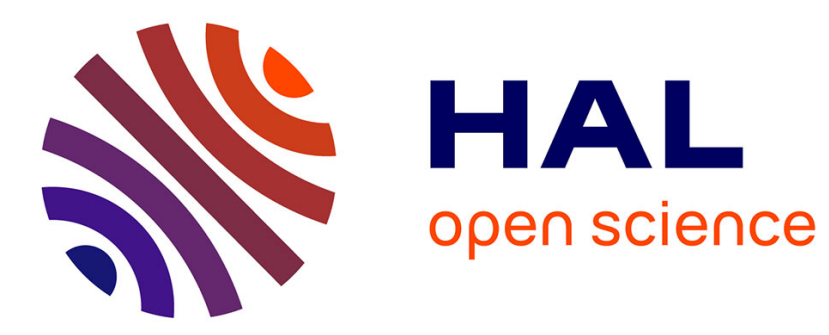

\title{
NEAR INFRARED SPECTROSCOPY USING GaAlAs-DIODE LASERS
}

\author{
H. Martin
}

\section{To cite this version:}

H. Martin. NEAR INFRARED SPECTROSCOPY USING GaAlAs-DIODE LASERS. Journal de Physique Colloques, 1987, 48 (C7), pp.C7-617-C7-620. 10.1051/jphyscol:19877149 . jpa-00226970

\section{HAL Id: jpa-00226970 https://hal.science/jpa-00226970}

Submitted on 1 Jan 1987

HAL is a multi-disciplinary open access archive for the deposit and dissemination of scientific research documents, whether they are published or not. The documents may come from teaching and research institutions in France or abroad, or from public or private research centers.
L'archive ouverte pluridisciplinaire HAL, est destinée au dépôt et à la diffusion de documents scientifiques de niveau recherche, publiés ou non, émanant des établissements d'enseignement et de recherche français ou étrangers, des laboratoires publics ou privés. 


\title{
NEAR INFRARED SPECTROSCOPY USING GaAIAS-DIODE LASERS
}

\author{
H. MARTIN \\ Institute of Physics, University of Stockholm, vanadisvägen 9 , \\ S-113 46 stockholm, Sweden
}

\begin{abstract}
Some spectroscopic techniques based on near infrared diode lasers are discussed with special emphasis on molecular spectroscopy. The semiconductor lasers provide for not only cheap coherent light sources of high quality, but also for new possibilities in experimental physics.
\end{abstract}

\section{INTRODUCTION}

The continuous development of the semiconductor lasers and their extension to the visible region is indeed very exciting for a variety of reasons. Certainly from a technical point of view - we all know of the different daily-life products and the accelerating importance of semiconductor lasers - but also from the point of view of natural science research. In this article the flavour of some of the characteristics of near infrared diode laser spectroscopy is given.

The diode laser was invented already in 1962, but these early diodes suffered from a large number of inconveniences. In the late seventies the situation drastically changed as the semiconductor technology had improved so as to achieve reliable operation. For instance, the lead-salt semiconductor lasers in the far infrared became commercially available. These lasers are the ones that one usually refers to when discussing molecular diode laser spectroscopy. A huge number of absorption experiments have been performed during the past eight years on a variety of molecular species using these lasers. They have to be cooled down to very low temperatures, though, and are incorporated in very sophisticated and expensive spectroscopic systems. The difficulties associated with these far infrared diode lasers are not at all present for the near infrared diode lasers which nowaday operate in room temperature.

The GaAlAs-diode lasers under discussion in this paper are characterized by single frequency operation, stable transverse and Iongitudinal mode structure, tenths of milliwatts $\mathrm{cW}$ optical power, less than $50 \mathrm{~mA}$ threshold current and high electrical to optical power conversion factor. They are continuously tunable at high resolution (better than $30 \mathrm{MHz}$ ) with tuning accomplished by changing the ambient temperature or drive current (Joule heating, which can be done very fast) and, in addition, they can be intensity and frequency modulated at several $\mathrm{GHz}$. The resolution can easily be improved one or two orders of magnitude by a simple optical feedback scheme using an external interferometer. The tuning range of each diode is approximately $13 \mathrm{~nm}$ for $0-50{ }^{\circ} \mathrm{C}$ operation but can be extended some $40 \mathrm{~nm}$ to shorter wavelength if sufficient cooling is applied. The main draw back of the simple GaAlAs-diode laser is the mode hops that occur now and then as the laser is tuned over wide spectral ranges. As a consequence spectral holes will appear in the output in a typical temperature scan, but most of the spectrum may be covered by scanning at different drive currents. The continuous tuning range is typically $5 \mathrm{~cm}^{-1}$ or $150 \mathrm{GHz}$. 
The production rate of GaAlAs-lasers is today around 2 million lasers a month. That makes them very cheap - in many cases less than 100 us dollars which of course makes them a very attractive device for generating coherent light. Laser spectroscopy no longer has to be expensive. Even the small scale researcher can now attain a more active place in mainstream physics and plan for multi-laser experiments, for instance. The simple engineering of diode lasers also provides for precise low-cost devices for diagnostics. A number of species like water-vapor, methane, nitric and carbon oxides, molecular nitrogen and oxygen all have absorption spectrum in the near infrared, as well as a large number of other diatomic molecules and atoms. A considerable number of atoms have already been examined using near infrared diode lasers [1]. In the following paragraphs a few experiments will briefly be discussed that demonstrates the potential of the diode lasers in molecular spectroscopy in both absorption and emission.

\section{VELOCITY-MODULATED ABSORPTION}

The velocity-modulated molecular ion spectroscopy was recently developed by Saykally and coworkers [2] in order to gain information of charged molecules in discharges. For molecular ions the spectrum of interest usually is hidden behind the spectrum of more abundant neutral molecules also present in the discharge. The method is based on the fact that charged molecules will be accelerated in the electric field of an ac discharge whereas neutrals will in a first approximation be unaffected. Hence, the ions will experience an alternating Doppler shift. With two laser beams collinear with the electric field - one inside and one outside the discharge tube - the differential absorption is detected by two PIN-diodes and analyzed by phase-sensitive amplifiers. As a result the $\mathrm{N}_{2}^{+}$spectrum shown in Fig.1 was obtained [3] where the ions are revealed by the derivative line-profile. By analyzing the signals at twice of this frequency one gets a spectrum which consists of the average of the total absorption when the polarity is switched. There we can simultaneuously monitor the neutral species and compare the two spectra. The supression of the neutrals in the lower spectrum is in this case roughly a factor of 50 .

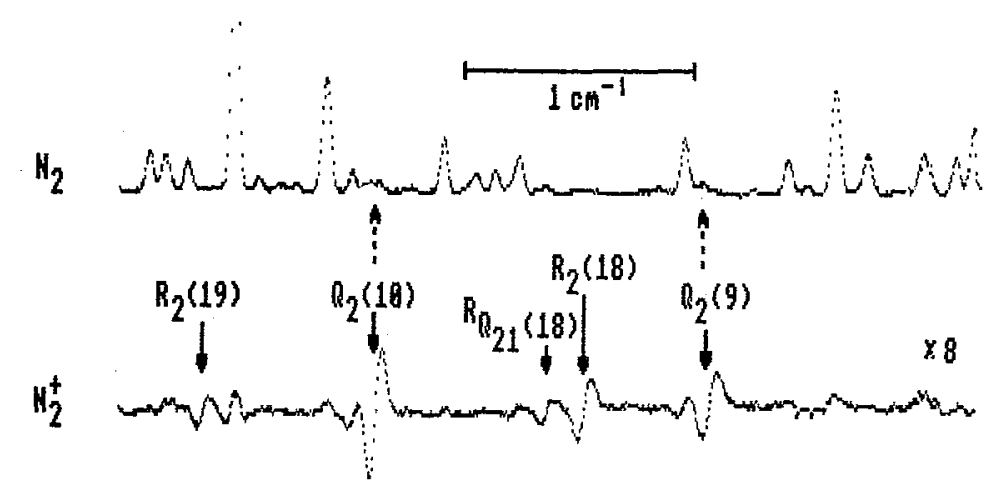

Fig.1 Part of a velocity-modulated diode laser spectrum of $\mathrm{N}_{2}^{+}$(lower trace) and a simultaneous recording of neutral $\mathrm{N}_{2}$ (upper trace) in the $785 \mathrm{~nm}$ region.

\section{FM SPECTROSCOPY}

The absorption in the $\mathrm{N}_{2}^{+}$lines in Fig.1 is of the order of a few parts of a million. If one still is not satisfied regarding the absorption sensitivity one can easily apply the very sensitive technique known as frequency modulated spectroscopy with heterodyne detection [4]. When dealing with Doppler or 
pressure broadened lines, a two-tone frequency modulation is needed as demonstrated by Gallagher on water vapor [5]. The two-tone approach was made since the optimum modulation frequency. normally should be larger than the linewidth which in this case is a few $\mathrm{GHz}$. At these high frequencies, however, low-cost detector devices have a poor response. The trick is then to apply still another modulation frequency determined by what frequency the detection system can manage, typically $10 \mathrm{MHz}$. In that way the sensitive heterodyne beat signal of the modulation sidebands will appear also at this lower frequency and impressive signal-to-noise ratios may be obtained - three orders of magnitude better than with simple modulation techniques.

\section{EXCITATION SPECTROSCOPY}

One example of emission experiments involving the GaAlAs-diode laser is given in the rotational analysis of the $\mathrm{B}^{2} \Sigma^{+}-\mathrm{X}^{2} \Sigma^{+}$transition in gaseous BaCl. Some 5000 rotational lines were collected [6]. A diode laser excitation spectrum was obtained by monitoring the absorption lines by detecting the subsequent laserinduced luminescence. This luminescence was passed through a small monochromator acting as an optical bandpass filter which allowed for selecting one vibrational transition and suppress the others. As a result overlapping spectral features were removed and the analysis was simplified. The diode laser was also used to calibrate a Fourier transform absorption spectrum by calibrating some Bacl lines accurately against neon reference lines. The neon lines were obtained using the optogalvanic effect readilly observed in a neon hollow cathode when a portion of the diode laser was directed into it.

\section{OPTICAL-OPTICAL DOUBLE-RESONANCE SPECTROSCOPY}

The optical power available nowadays from the near infrared diode lasers also make them attractive light sources for different kinds of double-resonance experiments. For instance, one suggestion is to pulse a diode laser at the Rabi frequency of a particular transition and take advantage of the transient population inversion for efficient stepwise excitation of high-lying Rydberg levels [1]. We (T. Gustavsson, H. Martin and P. Royen) have made a rotational analysis of the previously unknown $\mathrm{F}^{2 \Sigma^{+}}$state in BaCl by means of opticaloptical double-resonance, using a $\mathrm{cw}$ diode laser to populate selected quantum levels in the intermediate $B$ state. These were further excited to the $F$ state by a scanning dye laser. The F-B sub-Doppler resonances were detected by monitoring the UV fluorescence. A total of 500 different rotational lines were included in the analysis of the $F$ state.

\section{TIME-RESOLVED SPECTROSCOPY}

The experiments mentioned so far could in principle have been carried out using other types of lasers - like dye lasers for instance. That is not the case for time-resolved spectroscopy. Since one can scan a considerable portion of a spectrum with a diode laser in less than a microsecond, there is a number of experiments that one can imagine involving transient species produced by, for instance, flash-discharge, photo-flash or laser-vaporization. In our laboratory we have measured the radiative lifetime of the $B$ state in BaCl by using high frequency tuning of the diode laser in combination with single photon detection and the delayed coincidence technique. The laser is tuned at high repetition frequency $(100 \mathrm{kHz})$ through the spectral region of interest. The time-correlated spectrum then reveals the exponential decay from each spectral line excited during the period of a couple of nanoseconds (see Fig.2). If one is concerned about the effects from previous excited levels one can drive the laser by a step function which produces a non-linear tuning rate. The slower tuning at the end 


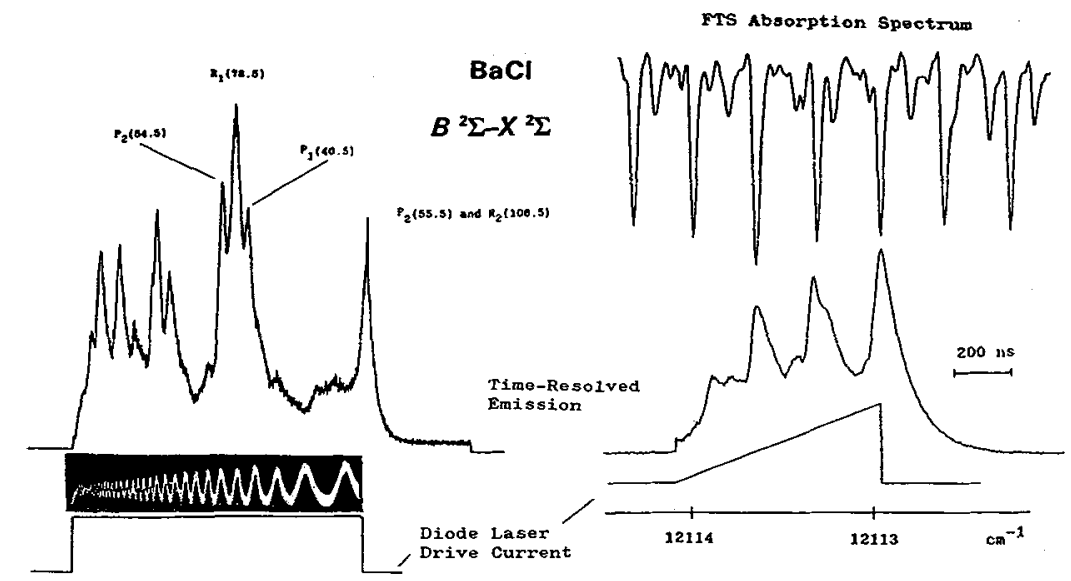

Fig.2 High frequency diode laser tuning and lifetime measurements on BaCl performed at the University of Stockholm by G. Gustafsson, H. Martin and P. Weijnitz.

of the scan makes the previously excited levels decay before the selected level is excited resulting in a pure exponential decay. Still, one obtains a portion of the spectrum in the multichannel analyzer which gives a documentation of what level one actually has measured the lifetime. By slightly adjusting the drive current dc level one can tune to the next spectral line of interest and start measuring again.

\section{THE FUTURE}

The future prospects regarding diode lasers for spectroscopic applications is very bright. Although there is at this moment a rapid development of tunable solid state lasers, nothing will beat the diode lasers regarding the price and engineering simplicity. The characteristics of the diode lasers are steadily improving. For instance, the distributed feedback lasers eliminate mode-jumps and the output powers of the single-element diode lasers are continuously increasing. 1 watt $\mathrm{cW}$ output power is already available from SONY corp. Quantum well lasers may offer the possibility of wide spectral tunability - in addition to ultralow threshold currents - and the available wavelength region for the diode lasers is indeed expected to extend considerably. NEC has announced their $680 \mathrm{~nm}$ laser to be available during 1988. The same company has demonstrated stable diode laser operation at $620 \mathrm{~nm}$ at $3^{\circ} \mathrm{C}$. Moreover, they have also demonstrated yellow laser output at $586 \mathrm{~nm}$ when cooled down to liquid nitrogen temperature. For shorter wavelengths, frequency doubling of the diode laser in optical fibers and possibly already in the diode laser cavity do indeed look very promising. Recent developments in non-linear optical crystal and fiber optics technology have made it possible to convert diode laser emissions with some tenths of milliwatts output powers to the violet with incredibly high conversion factors. As a result, diode laser sources will be commercially available soon covering the entire visible region in addition to the infrared. The exciting consequences of these developments are not hard to imagine.

\section{REFERENCES}

1. J.C. Camparo, Contemp. Phys. 26, 443 (1985).

2. C.S. Gudeman and R.J. Saykally, Ann. Rev. Phys. Chem. 35, 387 (1984).

3. B. Lindgren, H. Martin and U. Sassenberg, "Laser Spectroscopy VIII", Springer-Verlag, Berlin, 1987.

4. G.C. Bjorklund, Opt. Lett. 5,15 (1980).

5. T.F. Gallagher, "Laser Spectroscopy VII", Springer-Verlag, Berlin, 1987.

6. T. Gustavsson and H. Martin, Physica Scripta 34, 207 (1986). 